\title{
Association between ribotype and clinical form of enterocolitis with $C$.difficile in Western Romania: A 2-year study
}

\author{
ADELINA-RALUCA MARINESCU ${ }^{1}$, VOICHIT,A LĂZUREANU $^{1}$, RUXANDRA LAZA $^{1 *}$, \\ VIRGIL MUSTA $^{1 *}$, NARCISA NICOLESCU $^{1 *}$, TALIDA GEORGIANA CUT, $^{1 *}$, \\ CRISTINA DEHELEAN ${ }^{2}$, CRISTIAN OANCEA $^{1}$ and MONICA LICKER ${ }^{3}$ \\ ${ }^{1}$ Department XIII, ${ }^{2}$ Department II, ${ }^{3}$ Department XIV, 'Victor Babeș’ University \\ of Medicine and Pharmacy of Timisoara, 300041 Timisoara, Romania
}

Received July 23, 2021; Accepted August 24, 2021

DOI: $10.3892 / \mathrm{etm} .2021 .10944$

\begin{abstract}
During the last decade, several changes in the epidemiology, clinical symptoms, and evolution of Clostridium difficile infection (CDI) have been reported. The number of diagnosed cases has increased, especially in individuals over 60 years of age. There has also been an increase in the share of severe forms of the disease, the number of patients with recurrent infections and the lethality caused by this condition. The aim of the present study was to determine the incidence of CDI over the past few years; to monitor the bacterial toxin by ribotyping; to observe the migration of circulating toxins; to correlate the ribotyping with the clinical form of the disease; and to correlate the treatment with the ribotyping and the clinical form. Therefore, we performed an observational retrospective study regarding the incidence of CDI at 'Victor Babeș' Hospital of Infectious Diseases and Pneumophtisiology of Timişoara, between January 2016 and December 2017. The results revealed that 210 patients were hospitalized with a diagnosis of acute enterocolitis with $C$. difficile. All patients tested showed $C$. difficile toxin A/B positivity. In 28 cases, the ribotyping was positive with binary toxin and hypervirulent strain 027 . The tested patients were discharged or transferred from various medical/surgical clinics. In the study, we also ascertained the correlation between the clinical form of the disease and comorbidities and pre-hospital treatments as risk factors in the occurrence of the infection.
\end{abstract}

Correspondence to: Ms. Adelina-Raluca Marinescu or Dr Voichița Lăzureanu, Department XIII, 'Victor Babeș' University of Medicine and Pharmacy of Timisoara, 13 Gheorghe Adam Street, 300041 Timisoara, Romania

E-mail: marinescu_adelina24@yahoo.com

E-mail: vlazureanu@yahoo.com

*Contributed equally

Abbreviations: CDI, Clostridium difficile infection; IDSA, Infectious Diseases Society of America

Key words: ribotype, Clostridium difficile, treatment, clinical form, incidence
In accordance with the rise of CDI during the last decade, it was possible to observe the increase in the number of infectious recurrences. From this point of view, highlighting of the ribotype in this pathology becomes a primary aspect both by increasing the success rate in curing the disease (with a reduction in the risk of recurrence) and by targeted and non-empirical administration of the antibiotic.

\section{Introduction}

Over the last two decades, there has been a dramatic change in the epidemiology of Clostridium difficile infection (CDI). A disease considered to be a side effect of antibiotic use, easy to treat, is now associated with outbreaks, with increased mortality and morbidity. The incidence of CDI in hospitalized patients has increased dramatically with more than 300,000 reported cases per year, with an increased mortality rate. In the USA, more than 12,800 deaths were reported in 2017 due to this pathology, adding CDI to the list of diseases of the century we live in (1).

The new epidemic of CDI has been defined by community outbreaks affecting people considered to be at low risk of developing the disease. Initially, until the year 2000, it was considered that ribotype BI/NAP1/027 did not represent a risk of toxicity until the Quebec epidemic. Subsequently, several US hospitals reported outbreaks of CDI ribotype BI/NAP1/027, with fulminant clinical symptoms (2). The $\mathrm{BI} / \mathrm{NAP} 1 / 027$ ribotype has certain unique characteristics that could explain why the severity associated with this strain has increased (3). From the ribotype point of view, CDI is a real 'chameleon' of infectious pathology; however, unfortunately, the overall power of knowledge about circulating toxins in different sections in the Western part of Romania today is an enigma due to the additional cost of the specificity test.

Another essential problem in this pathology is the fact that the $C$. difficile strain carries several mobile genetic elements (4), which are able to confer resistance genes to antibacterial medication. The use of broad-spectrum and ultra-broad-spectrum antibiotics has allowed the development of hypervirulent strains with a rate of therapeutic failure, mortality and recurrence. Generally, most circulating strains are sensitive to metronidazole and vancomycin, although 
antimicrobial susceptibility testing has shown, in several international studies (5), lower susceptibility to metronidazole in infection with CD 027/BI/NAP1 strains, as well as with ribotypes 106 and 001 . Albeit the current protocols used in our country recommend this type of therapy in case of recurrence (6); the importance of ribotyping and targeted antibiotic therapy based on the antibiogram is thus emphasized once again.

A study conducted in 2013-2014, a collaboration among the Clinical Hospital for Infectious Diseases of Timisoara, the Laboratory of Microbiology, Timisoara County Emergency Clinical Hospital and the Discipline of Epidemiology, 'Victor Babeș' University of Medicine and Pharmacy of Timisoara, also demonstrated the prevalence of CDI endemicity in the western part of Romania, with an incidence of 20.57/15.70 per 1,000 patients discharged in $2013 / 2014$ or $17.73 / 14.04$ per 10,000 patients per day. As a result of this research, the incidence of the pathology could be estimated and the risk factors were investigated in relapses and cases with fulminant symptoms (7).

The objectives of this study were to determine the incidence of $C$. difficile infection within the past few years, to monitor bacterial toxin by ribotyping, to test circulating toxins, to correlate the ribotyping with the clinical form of the disease and to correlate the treatment with ribotyping and clinical form.

\section{Materials and methods}

Study population. We performed an observational retrospective study regarding the incidence of CDI at 'Victor Babes' Hospital of Infectious Diseases and Pneumophtisiology of Timisoara, between January 2016 and December 2017.

Upon admission, the patients signed the standardized informed consent by which they consented to their data being used for research purposes. The study was approved by the Ethics Committee of 'Victor Babeș' Hospital of Infectious Diseases and Pneumophtisiology of Timisoara.

'Victor Babeş' Hospital of Infectious Diseases and Pneumophtisiology of Timisoara is composed of two sections of infectious diseases, with a case rate of 2,076 cases in 2016 and 2,048 cases in 2017 (patients over 18 years of age).

Methods. We only included laboratory confirmed cases in patients aged 18 or older, subdivided in 6 age groups, regardless of sex, personal history, and probable infection source nosocomial or community acquired.

We collected demographic data, the presence of various comorbidities (malignancy, diabetes, chronic renal failure, cardiac, pulmonary, mild/moderate/severe liver pathology, or peripheral vascular, cerebrovascular, hematological diseases, dementia, gastro-duodenal ulcer, presence of concomitant infections); possible causes of immunosuppression during the last 2 months prior to the onset (chemotherapy, radiotherapy, corticosteroids, chronic dialysis, surgery including the type of intervention); other risk factors (inflammatory bowel disease, colorectal cancer, previous exposure to antimicrobial agents, enteral/parenteral nutrition); and clinical data.

The stool samples were collected through regular methods, using sterile recipients.
The etiology was confirmed by VIDAS ${ }^{\circledR} C$. difficile Toxin A \& B (bioMérieux) test, an ELFA (enzyme-linked fluorescent assay) that detects toxins A and B in fresh stool samples with a sensitivity of $88.3 \%$ and a specificity of $99.8 \%$. Ribotyping was performed using $C$. difficile assay polymerase chain reaction, which allowed the differentiation between toxin $B$ and binary toxin as well as the presumptive detection of strain $027 / \mathrm{NAP} / \mathrm{B} 1$ with a sensitivity of $93.49 \%$ and a specificity of $94.02 \%$.

Statistical analysis. Data analysis was performed using the Statistical Package for Social Sciences (SPSS) v.25 (IBM Corp.). We presented continuous variables as mean and standard deviation and categorical variables as frequency and percentages. For the evaluation of the potential connection between ribotype and clinical form of CDI, we employed the $\chi^{2}$ test. In order to determine the association between the treatment, clinical form and ribotype, a regression model and the Hosmer-Lemeshow test was used. P-value $<0.05$ was considered to indicate a statistically significant difference.

\section{Results}

Between January 2016 and December 2017, 210 patients were hospitalized with a diagnosis of acute enterocolitis with C. difficile. All patients tested showed $C$. difficile toxin A/B positivity.

In 2016, 95 patients with the diagnosis of $C$. difficile enterocolitis were hospitalized. If we refer to 2017, we can see an increase in 20 hospitalized cases with the same pathology, amounting to 115 reported cases (Table I).

The incidence of patients with $C$. difficile in the period of 2016-2017 was $\mathrm{I}_{\text {total }}=4.801164$. In 2016, the incidence was $\mathrm{I}_{2016}=4.190751$, and in $2017, \mathrm{I}_{2017}=5.419922$, there was an increase in the incidence of CDI comparing the years 2016 and 2017.

In this study, we followed the evolution of 210 patients, aged between 28 and 90 years, mean age $67.99 \pm 17.50$ years $(\mathrm{P}<0.0001)$. Regarding the distribution by sex, both in 2016 and in 2017, the female sex predominated, with a total number of 121 cases, divided into 53 cases (56\%), recorded in 2016 vs. 68 cases $(59 \%)$ in $2017(\mathrm{P}<0.0001)$. Conversely, the male sex maintained a linear pattern with 42 cases $(44 \%)$ reported in 2016 vs. 47 cases $(41 \%)$ in $2017(\mathrm{P}<0.0001)$ (Fig. 1).

Urban-rural distribution was found to have the same increased incidence (59\%) in urban areas (Utot) in 2016/2017, thus reporting 124 cases out of the total $(\mathrm{P}=0.0091)$. Regarding the rural environment (Rtot), it was found to have an equally increasing trend, with a percentage of $41 \%$ of the cases (86 patients), explainable due to easy access to the urban health system and the lack of territorial care systems (Table II).

The incidence of these infections was significantly higher in the age group over 61 years (totaling over half of the cases, $\mathrm{P}<0.0001$ ), followed by adults (40-60 years, over $20 \%$ of cases, $\mathrm{P}<0.0001)$. Although it was the prerogative of the study to research the elderly in the period of the study, there were also cases of young individuals under 40 (9.47\% in 2016, 5.20\% in 2017, $\mathrm{P}<0.0001$ ) (Table III).

Because most hospital-acquired CDI cases occur in elderly patients with an increased burden of chronic comorbidities 
Table I. Incidence of Clostridium difficile infection (CDI) at 'Victor Babeș' hospital of infectious diseases and pneumophtisiology of Timisoara.

\begin{tabular}{lcccc}
\hline Year & Hospitalized patients & Relapses & New cases & Total Clinic I + Clinic II \\
\hline 2016 & 95 & 8 & 87 & 2,076 \\
2017 & 115 & 4 & 111 & 2,048 \\
Total & 210 & 12 & 198 & 4,124 \\
\hline
\end{tabular}

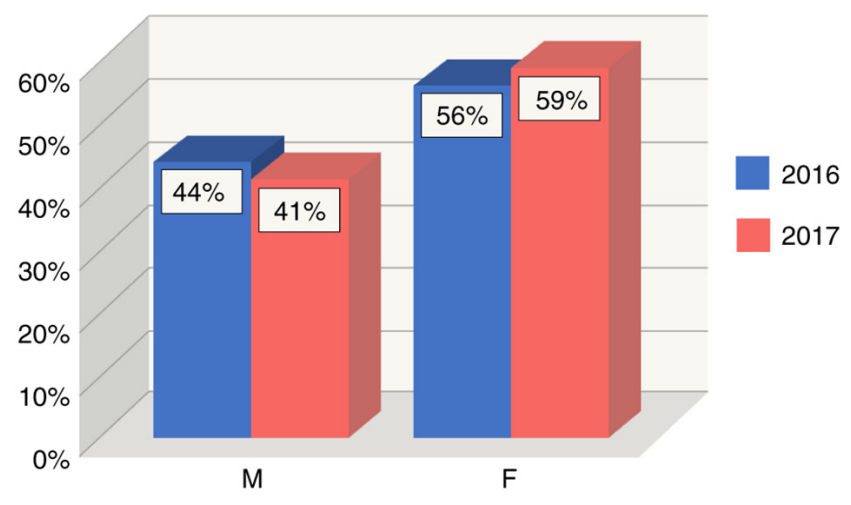

Figure 1. Sex distribution of the Clostridium difficile infection (CDI) cases. F, female; M, male.

affecting multiple organs and systems (8), multimorbidity itself may play a relevant role in defining CDI risk.

Comorbidity was well represented. Cardiovascular diseases were found to be a primary comorbidity (over 60\% of cases reported in both years, $\mathrm{P}=0.0018$ ), followed by renal pathology, metabolic diseases (diabetes), chronic lung disease and dementia (with a percentage of over $10 \%$ of cases in 2016 , $\mathrm{P}<0.0001$ ) (Table IV).

Because elderly patients are currently the ones who most frequently develop CDI, further studies are needed in order to explore the association between this infection and the areas of comorbidity, deficiencies and polymedication, which are intrinsic features of hospital-admitted geriatric patients.

The comorbidity score, Cumulative Illness Rating Scale (CIRS) can be a useful tool in CDI to estimate this additional risk, especially in patients undergoing long-term antibiotic treatment (9).

Until recently, septic shock was considered to be composed of three components: systemic hypotension, tissue hypoperfusion associated with organ dysfunction and hyperlactatemia $(10,11)$.

According to the new definition, septic shock can be diagnosed in two conditions. The first condition is persistent hypotension after fluid correction; it requires vasopressors to maintain mean blood pressure $>65 \mathrm{mmHg}$ (12). The second condition is the level of serum lactate $>2 \mathrm{mmol} / \mathrm{l}$. Since heart rate, respiratory rate and other laboratory data are not included, the diagnosis and recognition of septic shock have become simplified (13). This very new definition implies that elevated serum lactate levels may represent tissue hypoperfusion associated with signs of organ dysfunction in critically ill patients (14). In addition, it should be noted that the serum lactate level was decreased from 4 to $2 \mathrm{mmol} / \mathrm{l}$.

The level of serum lactate as a clinical tool was described about half a century ago by Broder and Weil (15). At that time, a serum lactate level $>4 \mathrm{mmol} / \mathrm{l}$ was associated with shock. Because serum lactate levels have been reduced to $2 \mathrm{mmol} / \mathrm{l}$, serum lactate levels are a more sensitive marker for septic shock.

Lactate is an important source of energy, especially during diet (when the body has no other resources). Therefore, when lactate is not produced, humans cannot survive. Lactate also contributes to the acidic environment by converting to lactic acid. Next, lactate is converted to bicarbonate, and it becomes a major source of alkalization of the body under normal conditions. Lactate of 1.4-1.5 mmol/day consists of the reduction of pyruvate, which is largely generated by anaerobic glycolysis. In tissue hypoxia, lactate is overproduced by increasing anaerobic glycolysis (16). Usually, lactic acid clearance occurs in the liver (60\%), followed by the kidneys (30\%) and to a lesser extent by other organs (heart and skeletal muscle).

Lactic acid clearance cannot exceed lactate production and can be altered during the critical state. Septic shock with liver dysfunction and acute kidney damage increase lactate levels due to decreased clearance.

Some patients recovering from septic shock have normal serum lactate values, although vasopressors are still needed to maintain an average blood pressure of $65 \mathrm{mmHg}$ or higher (17). In addition, low or normalized lactate levels are important signs of recovery from septic shock.

Within the case study in hospitalized patients in the period of 2016-2017, an increased prevalence of lactic acid was observed. A total of 105 cases (prevalence 0.5 ) showed a lactic acid value between $2-5 \mathrm{mmol} / \mathrm{l}$. Nine cases (prevalence 0.04 ) out of the 201 presented a lactic acid with a value $>5 \mathrm{mmol} / \mathrm{l}$ (Table $\mathrm{V}$ ).

If we correlate the ribotype with the clinical form of the disease by applying the $\chi^{2}$ test to see the association of the two variables, we obtained $\chi^{2}=5.9$ with $\mathrm{n}=2$ degrees of freedom, and $\mathrm{P}$-value $=0.0522>0.05$, which is why no conclusion can be drawn regarding the association of the two variables without the Cramer's correction $\mathrm{V}=0.167$, which indicates a weak association. In conclusion, it can be stated that the severity of the disease depends on the form of the ribotype (Table VI).

In terms of treatment, metronidazole in the form of oral tablets should be limited only to mild cases of the disease. Oral vancomycin remains the recommended treatment for mild/moderate forms that do not respond to metronidazole treatment. Repeated courses of metronidazole should be avoided due to the increased risk of potentially irreversible cumulative neurotoxicity. 
Table II. Distribution by urban vs. rural origin of the Clostridium difficile infection (CDI) cases for 2016 and 2017.

\begin{tabular}{llllllllll}
\hline Urban (U) distribution & Utot $=$ & 0.585714 & $59 \%$ & U2016= & 0.568421 & $57 \%$ & U2017= & 0.6 & $60 \%$ \\
\hline Rural (R) distribution & Rtot $=$ & 0.414286 & $41 \%$ & R2016= & 0.431579 & $43 \%$ & R2017 $=$ & 0.4 & $40 \%$ \\
\hline
\end{tabular}

Table III. Breakdown of the incidence of Clostridium difficile infection (CDI) by age group.

\begin{tabular}{lcccccc}
\hline & \multicolumn{5}{c}{ Age group (years) } \\
\cline { 2 - 7 } Year & $<40(\%)$ & $(40-50)(\%)$ & $(50-60)(\%)$ & $(60-70)(\%)$ & $(70-80)(\%)$ & $(80-90)(\%)$ \\
\hline 2016 & $9.47(9$ cases $)$ & $1.05(1$ case $)$ & $11.58(11$ cases $)$ & $17.90(17$ cases $)$ & $42.10(40$ cases $)$ & $17.90(17$ cases $)$ \\
2017 & $5.20(6$ cases $)$ & $5.20(6$ cases $)$ & $13.05(15$ cases $)$ & $17.40(20$ cases $)$ & $46.10(53$ cases $)$ & $13.05(15$ cases $)$ \\
\hline
\end{tabular}

Table IV. Comorbidities of the Clostridium difficile infection (CDI) cases.

\begin{tabular}{lccc}
\hline & \multicolumn{3}{c}{ Year } \\
\cline { 2 - 4 } Comorbidities & $2016(\%)$ & $2017(\%)$ & Total $(\%)$ \\
\hline Acute renal pathology & $17.89(17$ cases $)$ & $29.57(34$ cases $)$ & $47.46(51$ cases $)$ \\
Vascular cerebral pathology & $10.53(10$ cases $)$ & $4.35(5$ cases $)$ & $14.88(15$ cases $)$ \\
Malignant pathology & $10.53(10$ cases $)$ & $10.43(12$ cases $)$ & $20.96(22$ cases $)$ \\
Diabetes & $28.42(27$ cases $)$ & $21.74(25$ cases $)$ & $50.16(52$ cases $)$ \\
Chronic kidney failure & $14.74(14$ cases $)$ & $28.70(33$ cases $)$ & $43.44(47$ cases $)$ \\
Chronic heart disease & $60.00(57$ cases $)$ & $65.22(75$ cases $)$ & $125.22(132$ cases $)$ \\
Peripheral vascular disease & $5.26(5$ cases $)$ & $5.22(6$ cases $)$ & $10.48(11$ cases $)$ \\
Chronic lung disease & $13.68(13$ cases $)$ & $6.96(8$ cases $)$ & $20.64(21$ cases $)$ \\
Medium/severe hepatic pathology & $7.37(7$ cases $)$ & $9.57(11$ cases $)$ & $16.94(18$ cases $)$ \\
Dementia & $12.63(12$ cases $)$ & $8.70(10$ cases $)$ & $21.33(22$ cases $)$ \\
\hline
\end{tabular}

Table V. Lactic acid levels in the Clostridium difficile infection (CDI) cases.

\begin{tabular}{lccc}
\hline & \multicolumn{3}{c}{ Lactic acid } \\
\cline { 2 - 4 } Year & $<2 \mathrm{mmol} / \mathrm{l}$ & $2-5 \mathrm{mmol} / \mathrm{l}$ & $>5 \mathrm{mmol} / \mathrm{l}$ \\
\hline 2016 & 0.463157895 & 0.515789474 & 0.021052632 \\
2017 & 0.452173913 & 0.486956522 & 0.060869565 \\
Total & 0.457142857 & 0.500000000 & 0.042857143 \\
\hline
\end{tabular}

Although metronidazole may be associated with more common side effects and there has been a significant increase in treatment failure (especially in patients infected with emerging strain 027/BI/NAP1), oral metronidazole 500 three times a day for 10 days can be used to treat mild to moderate cases of CDI.

In current IDSA guidelines, metronidazole is used only in mild cases in patients without comorbidities or the risk of recurrence, as access to vancomycin or fidaxomicin is limited.
In a study published in 2015, a meta-analysis and systematic review was performed, comparing the efficacy and safety of metronidazole monotherapy with vancomycin monotherapy and combination therapy in patients with CDI. No statistically significant differences were found in the clinical cure rate between metronidazole and vancomycin for mild CDI form (4) or between monotherapy and combination therapy for CDI; however, the clinical cure rate was lower for metronidazole than for vancomycin for severe CDI.

In the present study, applying the $\chi^{2}$ test to see the association of the two variables (ribotype and treatment), we obtained $\chi^{2}=1.55$ with $n=2$ degrees of freedom, and $\mathrm{P}=0.46>0.05$, so the two variables are independent. The conclusion being that there is no association between the two variables (Table VII).

In order to determine the association between the 3 variables (treatment, clinical form and ribotype), the regression model was used, resulting in: $\operatorname{logit}(\mathrm{p})=-2.34-0.33 \mathrm{x}$ treatment $+0.68 \mathrm{x}$ form.

The Hosmer-Lemeshow test shows that the logistics model is the right one $(\mathrm{P}=0.6859>0.05)$ and that $86.67 \%$ of the cases were accurately predicted (Table VIII). 
Table VI. Association between disease form and ribotype of the Clostridium difficile infection (CDI) cases.

\begin{tabular}{lcccr}
\hline Disease form/Ribotype & Mild form & Average form & Severe form & Total \\
\hline 0.27 & 16 & 8 & 4 & 28 \\
Other & 130 & 45 & 7 & 182 \\
Total & 146 & 53 & 11 & 210 \\
\hline
\end{tabular}

Table VII. Association between ribotype and treatment of the Clostridium difficile infection (CDI) cases.

\begin{tabular}{|c|c|c|c|c|}
\hline Treatment/Ribotype & Metronidazole & Vancomycine & Metronidazole + vancomycine & Total \\
\hline 0.27 & 15 & 8 & 5 & 28 \\
\hline Other & 115 & 34 & 33 & 182 \\
\hline Total & 130 & 42 & 38 & 210 \\
\hline
\end{tabular}

Table VIII. Association of the ribotype with the clinical form of disease and treatment of the Clostridium difficile infection (CDI) cases.

\begin{tabular}{lccccccc}
\hline & \multicolumn{3}{c}{ Ribotype=0 } & & \multicolumn{3}{c}{ Ribotype=1 } \\
\cline { 2 - 4 } \cline { 6 - 8 } Form/Treatment & Metronidazole & Vancomycine & $\begin{array}{c}\text { Metronidazole }+ \\
\text { vancomycine }\end{array}$ & Metronidazole & Vancomycine & $\begin{array}{c}\text { Metronidazole }+ \\
\text { vancomycine }\end{array}$ \\
\hline Mild & 95 & 21 & 14 & & 13 & 2 & 0 \\
Average & 18 & 10 & 17 & & 1 & 5 & 2 \\
Severe & 2 & 3 & 2 & & 2 & 1 & 2 \\
\hline
\end{tabular}

\section{Discussion}

The etiology of infectious diarrhea is very varied, involving numerous pathogenic germs of bacterial, viral, parasitic, and fungal origin. The higher frequency represented by intestinal bacteriosis in the official statistics in our country does not represent the real incidence of the etiologies involved, but rather the concerns and limits of the activity of the specialized laboratory, especially for determining these bacterial etiologies.

The endemic nature of CDI reported in recent years cannot exclude the presence of deficiencies in the development of the epidemiological surveillance process of the hospital. In the presence of diseases with potential to induce secondary immunodeficiencies, the appropriate therapeutic act for the disease in question must be accompanied by the application of measures aimed at limiting nosocomial infections, in our case of Clostridium infection.

A study published in 2015 reported the cases of CDIs in our hospital. The incidence of infections in 2013-2014 was 20.57/15.70 per 1,000 patients discharged. If we compare it with current data, we can see an increase in the incidence of about $2 \%$ per hospitalized patient. This seeks to highlight once again the importance of the emergence of $C$. difficile infections (7).

Age is considered a primary risk factor for common forms of CDI (especially in young people, there are fewer relapses), but also for severe forms.
A feature that deserves a more detailed analysis is the role of general health condition, including dietary deficiency (hypoproteinemia) and the risk of CDI. Dietary deficiency (hypoproteinemia), the expression of biological aging, increases susceptibility to a variety of adverse events. Deficiency also results in increased exposure to health systems, including encounters with emergency departments, hospitalization and institutionalization, which has led to increased exposure to antibiotics, the most important risk factor for CDI.

The clinical picture of CDI causes a wide range of clinical manifestations, ranging from asymptomatic colonization of the gastrointestinal tract to diarrhea and colitis, which has the potential to progress to severe forms with sepsis, hemodynamic instability, and toxic dilation of the colon. This evolutionary method is associated with an increased risk of spontaneous perforation. A review of existing observational data has shown that $3 \%$ of patients with CDI will progress to fulminant colitis, which is associated with an $80 \%$ mortality rate.

Knowing the many factors that can contribute to the increased incidence of $C$. difficile infections in hospitals, it is necessary to intensify active surveillance, to promote the proper use of protective equipment and disinfection techniques, to ensure isolation spaces, to conduct visitor traffic control, to review antibiotic use policy, and to monitor measures undertaken in the case of 'serial' diseases. 


\section{Acknowledgements}

Professional editing, linguistic and technical assistance performed by Irina Radu, Individual Service Provider.

\section{Funding}

No funding was received.

\section{Availability of data and materials}

All data and materials supporting the results of the present study are available in the published article.

\section{Authors' contributions}

ARM and ML designed the study. VL, RL, VM, NN, CD and $\mathrm{CO}$ consulted the literature and collected the bibliographical data. ARM and ML wrote the paper. TGC and VL reviewed the data and edited the manuscript. All authors read and approved the final manuscript for publication.

\section{Ethics approval and consent to participate}

The study was approved by the Ethics Committee of 'Victor Babeș' Hospital of Infectious Diseases and Pneumophtisiology of Timisoara, Romania (approval number 4536/2021).

\section{Patient consent for publication}

Not applicable.

\section{Competing interest}

The authors declare that they have no competing interests.

\section{Authors' information}

ARM: MD PhD student, Infectious Disease Physician at Clinical ‘Dr. Victor Babeş’ Hospital for Infectious Diseases, Timisoara, Romania; PhD student at 'Victor Babeş' University of Medicine and Pharmacy. VL: MD PhD, Senior Lecturer of Infectious Diseases, Assistant Professor at the Infectious Diseases II Department, Member of the Advisory Commission of Infectious Diseases of the Ministry of Health, Romania. RL: MD PhD, Senior Lecturer of Infectious Diseases, Assistant Professor at the Infectious Diseases II Department of 'Victor Babeş' University of Medicine and Pharmacy, Timisoara, Romania. VM: MD PhD, Senior Lecturer of Infectious Diseases, Assistant Professor at the Infectious Diseases II Department of 'Victor Babeş' University of Medicine and Pharmacy, Timisoara, Romania. NN: MD PhD, Senior Lecturer of Infectious Diseases, Assistant Professor at the Infectious Diseases II Department of 'Victor Babeş' University of Medicine and Pharmacy, Timisoara, Romania. TGC: MD PhD student, Infectious Disease Physician, Clinical Hospital for Infectious Diseases 'Dr. Victor Babeș' Timisoara, Romania, PhD student at 'Victor Babeş' University of Medicine and Pharmacy. CD: CF PhD, Professor at the Toxicology and Drug Industry Department of 'Victor Babeş'
University of Medicine and Pharmacy CO: MD PhD, Professor at the Pneumology Department of 'Victor Babeş' University of Medicine and Pharmacy. ML: MD PhD, Professor at the Microbiology Department of 'Victor Babeş' University of Medicine and Pharmacy.

\section{References}

1. CDC: Antibiotic Resistance Threats in the United States 2019. CDC. 2019. Updated December 2019. http://www.cdc. gov/drugresistance/pdf/threats-report/2019-ar-threats-report-508. pdf. Accessed September 30, 2020.

2. Culligan EP and Sleator RD: Advances in the microbiome: Applications to Clostridium difficile infection. J Clin Med 5: 83, 2016.

3. Guh AY and Kutty PK: Clostridioides difficile infection. Ann Intern Med 169: ITC49-ITC64, 2018.

4. McDonald LC, Gerding DN, Johnson S, Bakken JS, Carroll KC, Coffin SE, Dubberke ER, Garey KW, Gould CV, Kelly C, et al: Clinical practice guidelines for Clostridium difficile infection in adults and children: 2017 update by the infectious diseases society of America (IDSA) and society for healthcare epidemiology of America (SHEA). Clin Infect Dis 66: e1-e 48, 2018.

5. Jump RLP and Donskey CJ: Clostridium difficile in the long-term care facility: Prevention and management. Curr Geriatr Rep 4: 60-69, 2015.

6. Willems RPJ, van Dijk K, Ket JCF and VandenbrouckeGrauls CMJE: Evaluation of the association between gastric acid suppression and risk of intestinal colonization with multidrug-resistant microorganisms: A systematic review and meta-analysis. JAMA Intern Med 180: 561-571, 2020.

7. Laza R, Jurac R, Crişan A, Lăzureanu V, Licker M, Popovici ED and Bădiţoiu LM: Clostridium difficile in western Romania: Unfavourable outcome predictors in a hospital for infectious diseases. BMC Infect Dis 15: 141, 2015.

8. Song JH and Kim YS: Recurrent Clostridium difficile infection: Risk factors, treatment, and prevention. Gut Liver 13: 16-24, 2019.

9. Knafl D, Vossen MG, Gerges C, Lobmeyr E, Karolyi M, Wagner L and Thalhammer F: Hypoalbuminemia as predictor of recurrence of Clostridium difficile infection. Wien Klin Wochenschr 131: 68-74, 2019.

10. Wei Y, Yang F, Wu Q, Gao J, Liu W, Liu C, Guo X, Suwal S, Kou Y,Zhang B, et al: Protective effects of bifidobacterial strains against toxigenic Clostridium difficile. Front Microbiol 9: 888, 2018.

11. Maisa A, Ross G, Verlander NQ, Fairley D, Bradley DT and Patterson L: Comparing the epidemiology of community- and hospital-associated Clostridium difficile infections in Northern Ireland, 2012-2016: A population data linkage and case-case study. Epidemiol Infect 147: e141, 2019.

12. Dharbhamulla N, Abdelhady A, Domadia M, Patel S, Gaughan J and Roy S: Risk factors associated with recurrent Clostridium difficile infection. J Clin Med Res 11: 1-6, 2019.

13. Appaneal HJ, Caffrey AR, Beganovic M, Avramovic S and LaPlante KL: Predictors of clostridioides difficile recurrence across a national cohort of veterans in outpatient, acute, and long-term care settings. Am J Health Syst Pharm 76: 581-590, 2019.

14. Chalhoub V, Kallab R, El Hajj A, Hachem K and Yazbeck P: Septic shock due to Clostridium tertium in an immunocompetent patient following colitis without inflammatory bowel disease. Anaesth Crit Care Pain Med 35: 167-168, 2016.

15. Linsenmeyer K, O'Brien W, Brecher SM, Strymish J, Rochman A, Itani K and Gupta K: Clostridium difficile screening for colonization during an outbreak setting. Clin Infect Dis 67: 1912-1914, 2018.

16. Magill SS, Edwards JR, Bamberg W, Beldavs ZG, Dumyati G, Kainer MA, Lynfield R, Maloney M, McAllister-Hollod L, Nadle J, et al: Multistate point-prevalence survey of health care-associated infections. N Engl J Med 370: 1198-1208, 2014.

17. Di Bella S, Ascenzi P, Siarakas S, Petrosillo N and di Masi A: Clostridium difficile toxins A and B: Insights into pathogenic properties and extraintestinal effects. Toxins (Basel) 8: 134, 2016. 